\title{
Cold atmospheric plasma destruction of solid proteins on stainless-steel surface and on real surgical instruments
}

\author{
Zerstörung fester Proteine auf Oberflächen chirurgischer Instrumente \\ aus Edelstahl unter Verwendung kalter Atmosphärendruck-Plasmen
}

\author{
Michael G. Kong ${ }^{1}$ \\ 1 Loughborough University, \\ Dept. of Electronic and \\ Electrical Engineerig, \\ Leicestershire, United \\ Kingdom
}

\section{Kurzfassung}

Die Möglichkeit der Abtötung von Mikroorganismen mit kalten atmosphärischen Plasmen ist bekannt. Ihre Fähigkeit, Proteine auf chirurgischem Instrumentarium zu zerstören, ist viel weniger aufgeklärt und es liegen nur wenige Studien dazu vor, obwohl chirurgische Instrumente typischerweise sowohl mit pathogenen Mikroorganismen als auch mit infektiösen Proteinen kontaminiert sind.

Mittels kalter atmosphärischer Heliumentladungen ist es möglich, Proteine zu zerstören. Als Arbeitsgas werden Helium-Sauerstoff-Mischungen bevorzugt, weil damit die Gastemperatur auf Raumtemperatur abgesenkt werden kann und so die Anwendung an polymeren Materialien möglich wird. Im Rahmen unserer Studie wurden zunächst Edelstahlplättchen als Testkörper zur Demonstration der plasmabasierten Proteinentfernung verwendet. Darüber hinaus wurden chirurgischen Zangen eingesetzt, die vorher bereits autoklaviert worden waren. Als Untersuchungsverfahren kamen die Laser-induzierte Fluoreszenzspektroskopie (LIF), die Rasterelektronenmikroskopie, die Elektronenenergiesdispersive Röntgenanalyse und die Elektrophorese zur Anwendung. Zielsetzung war der Nachweis, dass mit kalten atmosphärischen Plasmen die Zerstörung von Proteinen auf Oberflächen möglich ist, um damit die Leistungsfähigkeit, aber auch die Probleme einer Anwendung dieser Technologie für die Sterilisation aufzuzeigen.

In einer ergänzenden Studie wurden mögliche Mechanismen der Proteinzerstörung mittels optischer Emissionsspektroskopie und der Bestimmung der Kinetik der Proteinzerstörung aufgeklärt. Die Ergebnisse dieser Studie stützen die Auffassung, dass (1) kalte Atmosphärendruck-Plasmen in der Lage sind, sowohl Proteine zu zerstören als auch Mikroorganismen zu inaktivieren, (2) die Technologie zur Dekontamination chirurgischer Instrumente anwendbar ist und (3) atomarer Sauerstoff und angeregte Stickoxide die hauptsächlich wirksamen Spezies in diesen Prozessen sind.

\section{Corresponding author:}

Michael G. Kong

Loughborough University, Dept. of Electronic and Electrical Engineerig, Leicestershire LE11 3TU, United Kingdom M.G.Kong@lboro.ac.uk 


\section{Please cite as}

Kong MG. Cold atmospheric plasma destruction of solid proteins on stainless-steel surface and on real surgical instruments. GMS

Krankenhaushyg Interdiszip. 2008;3(1):Doc07.

This article is freely available from

http://www.egms.de/en/journals/dgkh/2008-3/dgkh000105.shtml

\section{Copyright}

(C)2008 Kong. This is an Open Access article distributed under the terms of the Creative Commons Attribution License

(http://creativecommons.org/licenses/by-nc-nd/3.0/deed.en). You are free: to Share - to copy, distribute and transmit the work, provided the original author and source are credited. 